\title{
Online Optimization Scheme with Dual-Mode Controller for Redundancy-Resolution with Torque Constraints
}

\author{
Jian Fang, Jianghai Zhao, Tao Mei and Jian Chen
}

\begin{abstract}
In this paper, an online optimization scheme is proposed for the real time path-tracking control of the redundant robot manipulator. In the proposed scheme, the inequality constraints are extended to the torque level to avoid the torque saturation. Besides, a dual-mode optimal controller is used to yield a feasible input during each control period, to resolve the solution space decreasing problem. Then the scheme is formulated as a Quadratic Program (QP), the computationally efficient Knitro-Based solver is applied to remedy the inescapable redundancy-resolution problem. Furthermore, the comparisons based on the different path tracking simulations between the proposed method and the velocity-level schemes demonstrate that the former is safer and more applicable. The experiment on a physical robot system further verifies the physical realizability of the proposed method.
\end{abstract}

Index Terms-Redundant Manipulator, Dual-Mode Optimal Controller, Online Optimization, Torque Constraints, Knitro-based QP Solver

\section{INTRODUCTION}

$\mathrm{T}$ he robot manipulator is redundant which means that it has more degrees of freedom (DOF) than required to achieve a prescribed task. The redundant DOF is normally used as the flexibility to avoiding obstacles or singularities, minimizing joint torques [1-3]. Path tracking planning is an appealing topic in the robotics field. The pseudo-inverse based method, including gradient projected method [4], extended Jacobian method [5], [6], augmented Jacobian method [7], [8] and weighted least norm method [9], can be used to solve this problem conveniently. These methods are generally used to implement some subtasks (secondary task), such as the singularity avoidance [10,11], torque optimization [12], and some multi-subtasks resolution [13, 14]. However, some physical limits of the redundant manipulator may be neglected in these methods, such as joint-velocity limits, jointacceleration limits, which may lead to a saturation and considerable path tracking errors, or even cause possible physical damage [15].

According to the literature published in the robotic field, online optimization method proposed a new perspective for resolving kinematics-redundancy problem during the recent decades $[15,16]$. Relatively, the online methods could be used to conquer the defects of the pseudo inverse based methods. In the online scheme, the equality and inequality constraints in the joint-velocity level $[17,18]$ or in the acceleration-level $[19,20]$ could be considered effectively. These schemes are generally configured as a quadratic program (QP) [20, 21]. The constraints may reflect the control strategy in our engineering application and the QP is converted to a nonlinear optimal problem, which is solved approximately by many solvers, such as numerical methods [22] and some types of neural networks based method [23], such as Zhang neural network (ZNN) solver and gradient neural network (GNN) solver [24].

As a brief summarization, the current online method mostly solved the redundancy-resolution problem on the velocity or acceleration level. The scheme for resolving kinematics in the velocity level probably introduces the discontinuity phenomenon during a continuous path tracking task [19]. This problem could be solved using the scheme in the acceleration-level, but the influence of gravity or the load of the end-effector is not taken into consideration effectively [20, 21]. On the other hand, the constraints of these schemes are established based on the kinematics model of the redundant robot. Nevertheless, they may not suitable for some path tracking task. For example, the dual-arm of the humanoid robot executes a path tracking task when the gravity of the arm and the load of the end-effector are considered. In this situation, the capacity or the driving ability of the joint actuator is the main limits for the task. It may lead the torque saturation for the control work if the capacity of the actuator is neglected. Fortunately, the physical quantity of the torque can be used to characterize this limit based on the robot dynamics.

Considering the engineering application of the humanoid arm, the constraints model must be extended to the torque level. Because the constraints in torque level synthetically consider the constraints in the joint velocity and acceleration level, which are stricter than that on the velocity or acceleration level. Obviously, the stricter constraints may decrease the solution space, which may cause some path tracking tasks unable to be finished. For another, the torque is computed based on the dynamics model; this may lead a heavy computation load when it is implemented in the engineering application, the current solvers based kinematics level may not suitable to this on dynamics level.

For extending the constraints to the torque level and solving its caused problem, a dual-mode controller is integrated to solve the redundancy-resolution problem in a limited space, and a Knitro instrument is applied in our work for resolving the redundancy-resolution in dynamics level firstly. Finally, the simulated and the experimental work are also presented in this paper to verify its efficiency. 
The remainder of the paper is organized as follows. Section II presents the formulation of the two online optimization schemes (resolution in velocity level and resolution in the acceleration level). Section III formulates the robot modeling method and robot control constraints. The dual-mode controller is presented in Section IV, which is followed by the simulation and experiment results in Section V. The conclusion and future work are given finally.

In this paper, a dual-mode online optimization scheme with torque constraints is proposed for the first time. The contribution of this paper is three-fold.

Firstly, the inequality constraints model is designed in the torque level for the redundancy-resolution, which synthetically considered the constraints in the joint velocity and acceleration level. It may formulate the predesigned physical limits more comprehensive and applicable.

Secondly, a dual-mode optimal controller is developed and incorporated to resolve the end effector tracking problem when the robot moved under a limited solution space.

Thirdly, corresponding to the computation based on the dynamic model, the efficient solver based on Knitro instrument is also first applied for redundancy-resolution in this paper.

\section{PRELIMINARY Formulation}

The relationship between the orientation/position vector $\mathbf{r}(\mathbf{t}) \in \mathfrak{R}^{m}$ of the end-effector in the Cartesian space and the joint angle vector $\boldsymbol{\theta}(\mathbf{t}) \in \mathfrak{R}^{n}$ in joint space could be depicted through a time varying equation:

$$
\mathbf{r}(\mathbf{t})=f(\boldsymbol{\theta}(\mathbf{t}))
$$

Due to the nonlinearity and redundancy of the mapping $f(\cdot)$, such a problem if often solved at the velocity or acceleration level. Differentiating (1) with respect to time $t$ yields the relationship between the Cartesian velocity $\dot{\mathbf{r}}(\mathbf{t})$ and the joint velocity $\dot{\boldsymbol{\theta}}(\mathbf{t})$

$$
\dot{\mathbf{r}}(\mathbf{t})=\mathbf{J}(\boldsymbol{\theta}(\mathbf{t})) \dot{\boldsymbol{\theta}}(\mathbf{t})
$$

Where $\mathbf{J}(\boldsymbol{\theta}(\mathbf{t}))=\partial f(\boldsymbol{\theta}(\mathbf{t})) / \partial \boldsymbol{\theta}(\mathbf{t}) \in \mathfrak{R}^{m \times n} \quad$ is the Jacobian matrix. Differentiating the (2) could further yield the relationship between the end-effector acceleration $\ddot{\mathbf{r}}(\mathbf{t})$ and the joint-acceleration $\ddot{\boldsymbol{\theta}}(\mathbf{t})$;

$$
\ddot{\mathbf{r}}(\mathbf{t})=\mathbf{J}(\boldsymbol{\theta}) \ddot{\boldsymbol{\theta}}+\dot{\mathbf{J}}(\boldsymbol{\theta}) \dot{\boldsymbol{\theta}}
$$

Where the $\dot{\mathbf{J}}(\boldsymbol{\theta})$ is the time derivative of the Jacobian matrix , the equation (1)-(3) depict the kinematics of the robot manipulator, and the solution for this method is infinite, because the robot manipulator is redundant degree of freedom, namely, the Jacobian matrix is not a square matrix.

The pseudo inverse-based method to the (2) and (3) can be formulated as two parts, namely, least-norm particular solution and homogeneous solution, like as equation (4),(5).

$$
\dot{\boldsymbol{\theta}}=\mathbf{P} \dot{\mathbf{r}}+(\mathbf{I}-\mathbf{P J}) \boldsymbol{\Phi}
$$

$$
\ddot{\boldsymbol{\theta}}=\mathbf{P} \ddot{\mathbf{r}}_{\mathrm{a}}+(\mathbf{I}-\mathbf{P J}) \Phi
$$

Where the $\mathbf{P}=\mathbf{J}^{\mathbf{T}}\left(\mathbf{J J}^{\mathrm{T}}\right)^{-1} \in \mathfrak{R}^{n \times m}$ denotes the pseudo inverse matrix of the $\mathbf{J}(\boldsymbol{\theta}), \ddot{\mathbf{r}}_{\mathbf{a}}=\ddot{\mathbf{r}}-\dot{\mathbf{J}}(\boldsymbol{\theta}) \dot{\boldsymbol{\theta}} \in \mathfrak{R}^{m}$, and $\boldsymbol{\Phi}$ is an arbitrary vector selected as gradients of some performance indices.

To better introduce our dual-mode optimal controller, the following redundancy-resolution schemes (with one at the velocity level and the other at the acceleration level) are presented, investigated, and formulated finally as solvable QPs, which could be applied in real-time motion control of the robot manipulators.

\section{A. Velocity-Level Redundancy-Resolution Scheme}

The normally used minimum-velocity-norm criterion [25, 26] has been widely adopted by most researchers in the velocity-level redundancy-resolution scheme, including an optimal objective and an equality constraint, as shown in the following:

$$
\begin{array}{lc}
\text { Minimize } & \frac{1}{2}\|\dot{\boldsymbol{\theta}}\|_{2}^{2} \\
\text { Subject to } & \mathbf{J}(\boldsymbol{\theta}) \dot{\boldsymbol{\theta}}=\dot{\mathbf{r}}
\end{array}
$$

Where $\|\cdot\|_{2}$ denotes the two-norm of a vector and (7) relates to the end-effector's primary task in task space. The $\|\dot{\boldsymbol{\theta}}\|_{2}^{2} / 2=$ $\dot{\boldsymbol{\theta}}^{\mathrm{T}} \dot{\boldsymbol{\theta}} / 2$, and superscript " $\mathrm{T}$ " denotes the transposes of a vector. Generally, the scheme (6), (7) can be expressed as a time-varying QP problem that is subject to an equality constraint, as shown in the following:

$$
\begin{aligned}
& \text { Minimize } \frac{1}{2} \mathbf{x}^{\mathrm{T}} \mathbf{W} \mathbf{x}+\mathbf{q}^{\mathrm{T}} \mathbf{x} \\
& \text { Subject to } \mathbf{C x}=\mathbf{d}
\end{aligned}
$$

Where $\mathbf{x}=\dot{\boldsymbol{\theta}} \in \mathfrak{R}^{n}, \mathbf{W}=\mathbf{I} \in \mathfrak{R}^{n \times n}$ denote the identity matrix, $\mathbf{C}=\mathbf{J} \in \mathfrak{R}^{m \times n}, \mathbf{d}=\dot{\mathbf{r}} \in \mathfrak{R}^{m}$, and $\mathbf{q}=\mathbf{0} \in R^{m}$.

\section{B. Acceleration level Redundancy-Resolution scheme}

Similarity, the redundancy resolution in the acceleration level can be depicted in the following:

$$
\begin{array}{ll}
\text { Minimize } & (\ddot{\boldsymbol{\theta}}+\mathbf{p})^{\mathrm{T}}(\ddot{\boldsymbol{\theta}}+\mathbf{p}) / 2 \\
\text { Subject to } & \mathbf{J}(\boldsymbol{\theta}) \ddot{\boldsymbol{\theta}}=\mathbf{r}_{\mathbf{a}}
\end{array}
$$

Where $\mathbf{p}=\lambda \dot{\boldsymbol{\theta}}$, and the parameter $\lambda>0$ should be large enough. Thus, the scheme (10) and (11) also could be concluded as a QP formulation shown in the following,

$$
\begin{aligned}
& \text { Minimize } \frac{1}{2} \mathbf{x}^{\mathrm{T}} \mathbf{W} \mathbf{x}+\mathbf{q}^{\mathrm{T}} \mathbf{x} \\
& \text { Subject to } \mathbf{C x}=\mathbf{d}
\end{aligned}
$$

The (12) and (13) is similar to the (8) and (9), nevertheless, in the acceleration scheme, $\quad \mathbf{x}=\ddot{\boldsymbol{\theta}} \in \mathfrak{R}^{n} \quad, \quad \mathbf{W}=\mathbf{I} \in \mathfrak{R}^{n \times n} \quad$, $\mathbf{C}=\mathbf{J} \in \mathfrak{R}^{m \times n}, \mathbf{d}=\ddot{\mathbf{r}}_{\mathrm{a}} \in \mathfrak{R}^{m}$ and $\mathbf{q}=\lambda \dot{\boldsymbol{\theta}} \in \mathfrak{R}^{n}$. The scheme (10) and (11) solved the redundancy-resolution problem from the aspect of the acceleration level. This scheme can provide a new prospective to control the manipulator. 


\section{DuAl Mode ONLINE OPTIMIZATION METHOD}

As aforementioned, the redundancy-resolution problem solved using optimization techniques at velocity or acceleration level, have some weaknesses when we considered the joint torque. When the path tracking task is executed, an auxiliary proportional control law is designed for the tracking problem:

$$
\mathbf{u}=\dot{\mathbf{r}}+k_{p} \mathbf{x}_{\mathbf{n}}^{\mathbf{e}}
$$

Where the $\mathbf{x}_{\mathbf{n}}^{\mathrm{e}}=\mathbf{x}_{\mathrm{n}}^{\mathrm{d}}-\mathbf{x}_{\mathrm{n}}$ denotes the end-effector position tracking error, $k_{p}$ is the positive proportional feedback constants. This control law is set to implement the path tracking when the end-effector current position does not coincide to initial position of the desired path. Normally, by jointly actuating the motion to realize $\mathbf{u}$ (or described as $\mathbf{u}(\mathbf{t})$ ) at each interpolation cycle, the end-effector tracking problem can be resolved exponentially. Before the introduction of the dual-mode controller, the mechanical constraints of the joints are presented firstly.

\section{A. Mechanical Constraints in Torque Level}

We consider that each robot rotatory joint is subject to a set of identical joint rotation limit, angular speed, and angular acceleration, i.e., $\forall i=1,2, \cdots, \mathrm{n}$,

$$
\begin{gathered}
\boldsymbol{\theta}_{\text {min }} \leq \boldsymbol{\theta} \leq \boldsymbol{\theta}_{\text {max }} \\
\dot{\boldsymbol{\theta}}_{\text {min }} \leq \dot{\boldsymbol{\theta}} \leq \dot{\boldsymbol{\theta}}_{\text {max }} \\
\ddot{\boldsymbol{\theta}}_{\text {min }} \leq \ddot{\boldsymbol{\theta}} \leq \ddot{\boldsymbol{\theta}}_{\text {max }}
\end{gathered}
$$

The constraints (15)-(17) formulate the limitations in the velocity and acceleration level. To implement the fast continuous path tracking, the inescapable problem is the large acceleration and/or deceleration torque which is necessary for fast continuous path tracking control at its start position and its goal position. In this situation, the saturation avoidance of the joint torque must be considered. Considering the redundant robot dynamics based on the Euler-Lagrangian method, the dynamics of the redundant robot can be formulated and expressed using variable $\theta$, as shown in the following.

$$
\mathbf{H}(\boldsymbol{\theta}) \ddot{\boldsymbol{\theta}}+\mathbf{c}(\boldsymbol{\theta}, \dot{\boldsymbol{\theta}}) \dot{\boldsymbol{\theta}}+\mathbf{g}(\boldsymbol{\theta})=\boldsymbol{\tau}
$$

Where $\mathbf{H}(\boldsymbol{\theta})$ is the robot inertia matrix, $\mathbf{c}(\boldsymbol{\theta}, \dot{\boldsymbol{\theta}})$ is the Coriolis and centrifugal force vector, and $\mathbf{g}(\boldsymbol{\theta})$ is the vector for gravity force, respectively. $\boldsymbol{\tau}=\left(\tau_{1}, \tau_{2}, \ldots \tau_{n}\right)$ is the applied motor torque on $\boldsymbol{\theta}$. As we concerned the function of the dynamics, the joint torque is related by the joint angular acceleration and joint angular velocity. However, when the nonlinearity of the dynamics parameters (e.g. $\mathbf{H}(\boldsymbol{\theta})$ and $\mathbf{c}(\boldsymbol{\theta}, \dot{\boldsymbol{\theta}})$ ) are taken into consideration, the optimal scheme in the velocity level or in the acceleration level could not reflect the variation in the torque level directly. Therefore, a new constraint inequality in the torque level for limiting redundancy-resolution is shown in the following.

$$
\tau_{\min } \leq \boldsymbol{\tau} \leq \boldsymbol{\tau}_{\max }
$$

Where the $\tau_{\min }$ and the $\tau_{\max }$ denote the upper and the lower limits of the joint torque, respectively. Considering the dynamic function (18), the joint acceleration $\ddot{\boldsymbol{\theta}}$ at time $t$ may be approximated as:

$$
\ddot{\boldsymbol{\theta}}(\mathbf{t}) \approx \frac{1}{\Delta t}(\dot{\boldsymbol{\theta}}(\mathbf{t})-\dot{\boldsymbol{\theta}}(\mathbf{t}-\Delta \mathbf{t}))
$$

with $\dot{\boldsymbol{\theta}}(\mathbf{t})$ and the $\dot{\boldsymbol{\theta}}(\mathbf{t}-\Delta \mathbf{t})$ representing the $n \times 1$ joint rate vector at time $t$ and $t-\Delta t$, respectively. $\Delta t$ denotes the interpolation time. We rewrite the dynamic function as:

$$
\frac{\mathbf{H}(\boldsymbol{\theta})}{\Delta t}(\dot{\boldsymbol{\theta}}(\mathbf{t})-\dot{\boldsymbol{\theta}}(\mathbf{t}-\Delta \mathbf{t}))+\mathbf{c}=\boldsymbol{\tau}
$$

which $\mathbf{c}=\mathbf{c}(\boldsymbol{\theta}, \dot{\boldsymbol{\theta}}) \dot{\boldsymbol{\theta}}(\mathbf{t}-\Delta \mathbf{t})+\mathbf{g}(\boldsymbol{\theta})$ and substituting (21) into (19), the above joint torque inequality constraints can be written in matrix form:

$$
\mathbf{A} \dot{\boldsymbol{\theta}} \leq \mathbf{B}
$$

with $\mathbf{A}=\left[\begin{array}{c}\mathbf{H} \\ -\mathbf{H}\end{array}\right]_{2 n \times n}$

and $\mathbf{B}=\left[\begin{array}{c}\left(\boldsymbol{\tau}_{\max }-\mathbf{c}\right) \Delta t+\mathbf{H} \dot{\boldsymbol{\theta}}(\mathbf{t}-\Delta \mathbf{t}) \\ -\left(\boldsymbol{\tau}_{\min }-\mathbf{c}\right) \Delta t-\mathbf{H} \dot{\boldsymbol{\theta}}(\mathbf{t}-\Delta \mathbf{t})\end{array}\right]_{2 n \times 1}$

\section{B. Dual-mode optimal controller design}

The dual-mode controller is implemented based on two optimization schemes. Basically, we want to minimize the sum of joint angular deviations during each interpolation period $(t, t+\delta t)$. That is, design a nonlinear optimal criterion to minimize the 2-norm of $\dot{\boldsymbol{\theta}}(\mathbf{t}) \delta t$ as follows:

$$
\begin{aligned}
& \text { Minimize } \quad \ell_{1}=\|\dot{\boldsymbol{\theta}}(\mathbf{t}) \boldsymbol{\delta} \mathbf{t}\|_{2} \\
& \text { Subject to } \mathbf{J}(\mathbf{t}) \dot{\boldsymbol{\theta}}(\mathbf{t})=\mathbf{u}(\mathbf{t}) \\
& \text { and (22) }
\end{aligned}
$$

where (13) implies that the auxiliary PD input $\mathbf{u}(\mathbf{t})$ is realized by the actuating joints. However, as aforementioned, $\mathbf{u}(\mathbf{t})$ may be difficult to realize when the solution space is severely limited. Under such conditions, we want to maximize the end-effector tracking rate for fast tracking control, and thus design a nonlinear optimal criterion to maximize the effectiveness of the rotatory joints in driving the end-effector towards the direction of $u(t)$, i.e.

$$
\begin{aligned}
& \text { Maximize } \ell_{2}=[\mathbf{J}(\mathbf{t}) \dot{\boldsymbol{\theta}}(\mathbf{t})] \cdot \mathbf{u}(\mathbf{t}) \\
& \text { Subject to }[\mathbf{J}(\mathbf{t}) \dot{\boldsymbol{\theta}}(\mathbf{t})] \times \mathbf{u}(\mathbf{t})=\mathbf{0} \\
& \text { and (22) }
\end{aligned}
$$

where "." and " $x$ " denote the dot product and the cross product, respectively. It should be noted that using (26), we demand that the rotatory joints do not produce motion perpendicular to $\mathbf{u}(\mathbf{t})$. As know the infinity of the redundant kinematics solution, the optimal objective is used to acquire a joint angular vector $\dot{\boldsymbol{\theta}}(\mathbf{t})$ in joint space, which can be ensured the modulus value of $\mathbf{J}(\mathbf{t}) \dot{\boldsymbol{\theta}}(\mathbf{t})$ mostly approximates to the desired motion $\mathbf{u}(\mathbf{t})$. In other words, the constraint (26) is used to guarantee the direction of the solution vector, and the optimal objective (25) is used to maximize the modulus value of the solution vector. 
Remarks:

Firstly, in this dual mode controller, we choose the (22) instead of the (17) as the constraint because the torque can be applied to represent the motor driving ability. The saturation of the command torque can be avoided effectively using this constraint.

Secondly, the dual-mode controller switches between these dual modes intelligently. Normally, the redundancy-resolution is solved in first mode. When the solution space is limited, then the controller switches to the second mode to find feasible solution.

Thirdly, compared to the velocity and the acceleration level scheme aforementioned, the dynamics of the robot manipulator is composed to the constraint in the torque level. Therefore, the computation load for this scheme may increase heavily.

\section{The Knitro-based QP solver}

To solve the computation efficiency problem as aforementioned, the online scheme combined with dual-mode optimal controller can be reformulated as the following two QPs:

First QP:

$$
\begin{array}{ll}
\text { Minimize } & \left(\mathbf{x}^{\mathbf{T}} \mathbf{M x}\right) w^{2} / 2 \\
\text { Subject to } & \left\{\begin{array}{l}
\mathbf{L x}=\mathbf{U} \\
\mathbf{A x} \leq \mathbf{B}
\end{array}\right.
\end{array}
$$

Second QP:

$$
\begin{array}{ll}
\text { Maximize } & (\mathbf{L x}) \cdot \mathbf{U} \\
\text { Subject to } & \left\{\begin{array}{l}
(\mathbf{L x}) \times \mathbf{U}=\mathbf{0} \\
\mathbf{A X} \leq \mathbf{B}
\end{array}\right.
\end{array}
$$

where the $\mathbf{M}=\mathbf{I}, \mathbf{L}=\mathbf{J}(\mathbf{t}), \quad \mathbf{U}=\mathbf{u}(\mathbf{t}), \quad \mathbf{x}=\dot{\boldsymbol{\theta}}(\mathbf{t})$ and $w=\delta t$ is the constant span of the interpolation time.

In order to improve the computational efficiency, the Knitro instrument is used in our work, the Knitro optimization tool successfully integrates two nonlinear optimization approaches, namely, the interior-point approach and the active-set approach, in the solution search algorithm [27, 28]. In our work, the interface, $k \operatorname{trlink}()$, is used. The parameters of this interface are configured as the (27-30). $\mathbf{x}=\dot{\boldsymbol{\theta}}(\mathbf{t})$ is the variable of the scheme. When the first QP cannot find a suitable solution, the interface of the $k \operatorname{trlink}()$ will return a flag, which is less than zero. Then the first mode is aborted, the second mode is activated afterwards. If the second mode still cannot find a solution, the scheme will exit simultaneously, and return a flag which indexed the failure of the resolution. Depending on problem characteristics, Knitro intelligently switches between the interior point approach and the active-set approach to search for solutions, which not only enhances the robustness of the search algorithm, but also quickens the solution search. In addition, this instrument is implemented by using $\mathrm{C}$ language; it can be integrated into our engineering application conveniently.

\section{Simulations}

In this section, to validate the effectiveness of the proposed control strategy, computer simulations are performed based on a real-robot arm manipulator, named as Xing Tian (XT), which is originated from the ancient Mars of the Chinese. The XT is designed for participating DARPA (Defense Advanced Research Projects Agency) Robotics Challenge (DRC). Two redundant arm manipulators are designed for this robot to make it can be worked possibly like human. The physical parameters (e.g. gear ratio, torque limits) of the 7 joints used in the following simulations are shown in Table I. The end-effector of the robot manipulator is required to track two different paths: straight-line and circular path.

Table I. The joint related parameters

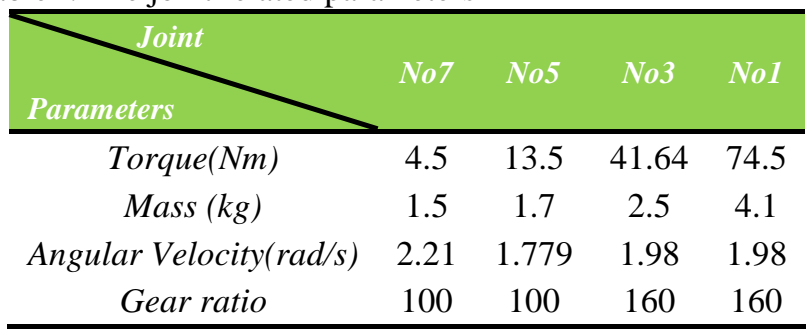

Remarks: the parameters shown in the table. I, are the real output parameters of the joint. In this table the joint 1 and joint 2 , joint 3 and joint 4 , joint 5 and joint 6 , have same parameters, respectively. These parameters are designed for a dual-arm of XT that it can pack a 4-5 kilogram of object. In Tab. I, the torque means the joint output moment, which is equal to the motor output moment multiply by the gear ratio. The gear ratio is also shown in last row of the Tab. I. Similarly, the angular velocity of the joint is the joint angular velocity, which is equal to the motor's velocity divided to their gear ratio.

\section{A. Straight-line path tracking}

In this section, the end-effector of the XT arm with physical limitations is expected to track a straight-line path. We exploit the cycloid function to plan the velocity of the end-effector in the Cartesian space, as shown in the following.

We calculate the derivation of the (31) and get the velocity function in the Cartesian space shown in the following:

$$
\left\{\begin{array}{l}
v(x)=\frac{d x}{T}-\frac{d x}{T} \cos (2 \pi t / T), \forall t \in[0, T] \\
v(y)=\frac{d y}{T}-\frac{d y}{T} \cos (2 \pi t / T), \forall t \in[0, T] \\
v(z)=\frac{d z}{T}-\frac{d z}{T} \cos (2 \pi t / T), \forall t \in[0, T]
\end{array}\right.
$$

Where the $v(x), v(y), v(z)$ denote the velocity in the $x, y$, $z$ direction, respectively. $d x, d y$ and $d z$ denote the distance between the start point and the end point in the $x, y$ and $z$ direction, respectively. The $T$ denotes the task duration time

In the computer simulation, the duration time for the straight line path tracking task is set as $2 \mathrm{~s}$ (i. e. $T=2 \mathrm{~s}$ ). In addition, the initial state for the joint space is set as [0.50 -0.0029 0.0164 $2.33530 .0001-2.3324-0.5879$ ] rad, namely, the initial position of the end-effector in the Cartesian space is around the point $[0.3,0.2,0.5]$ meter, during the task execution, we expected that the joint motion executed under the safe limitation of their torque parameters (e. g. $\tau^{ \pm}$).

Firstly, the kinematic resolution problem of XT manipulator is handled via Knitro instrument with velocity level constraints being considered but the torque constraint is 
neglected. The computer simulation results are shown in Fig. 1. The tracking path and the desired path of the end-effector are shown in the Fig. 1(a) with blue line and red line, respectively. It can illustrate the path tracking task executed well. From the Fig. 1(a), the vibration may be existed around the beginning of the path tracking task. This phenomenon can be illustrated from the Fig. 1(b), which depicts the tracking error of the end-effector in $x, y$ and $z$ three direction. We found that the errors in three directions are convergence to the zero, respectively. But it has some fluctuations at the beginning point of the path tracking. Furthermore, the curve characterized the joint angular velocity $\omega_{1}, \omega_{2} \ldots \omega_{6}, \omega_{7}$ are shown in the Fig. 2(a) with different color, respectively. Compared with the joint physical limits shown in Table I, the joint velocities joint-velocity limits have not been activated. Compared with the joint torque limits in Table I, however, the joint torque in the simulations reach their corresponding limits which is shown in the Fig. 2(b), (c), and (d). That is to say, the joint-torque over range phenomenon has happened. It is worth mentioning that this phenomenon is not desired in engineering applications because it may cause the vibration of the end-effector. This would thus lead to the robot instability of the control system. Because the posture of the end-effector is invariant, the torque of the last three joint (joint 5, joint 6 and joint 7) is little change. Considering the limitation of the paper length, the torque curves of these joints are not shown here.
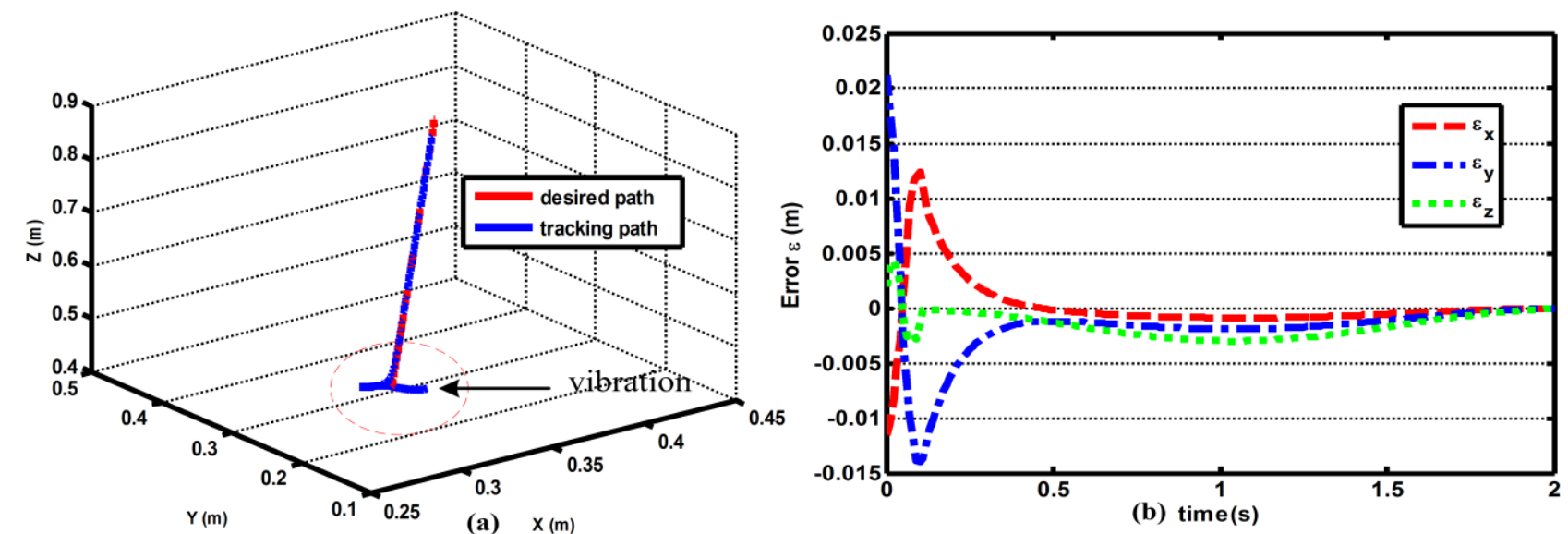

Fig.1. End-effector of the XT arm tracks a straight line path without considering our proposed scheme. (a) Tracking path and desired path. (b) Cartesian positioning error of the end-effector.
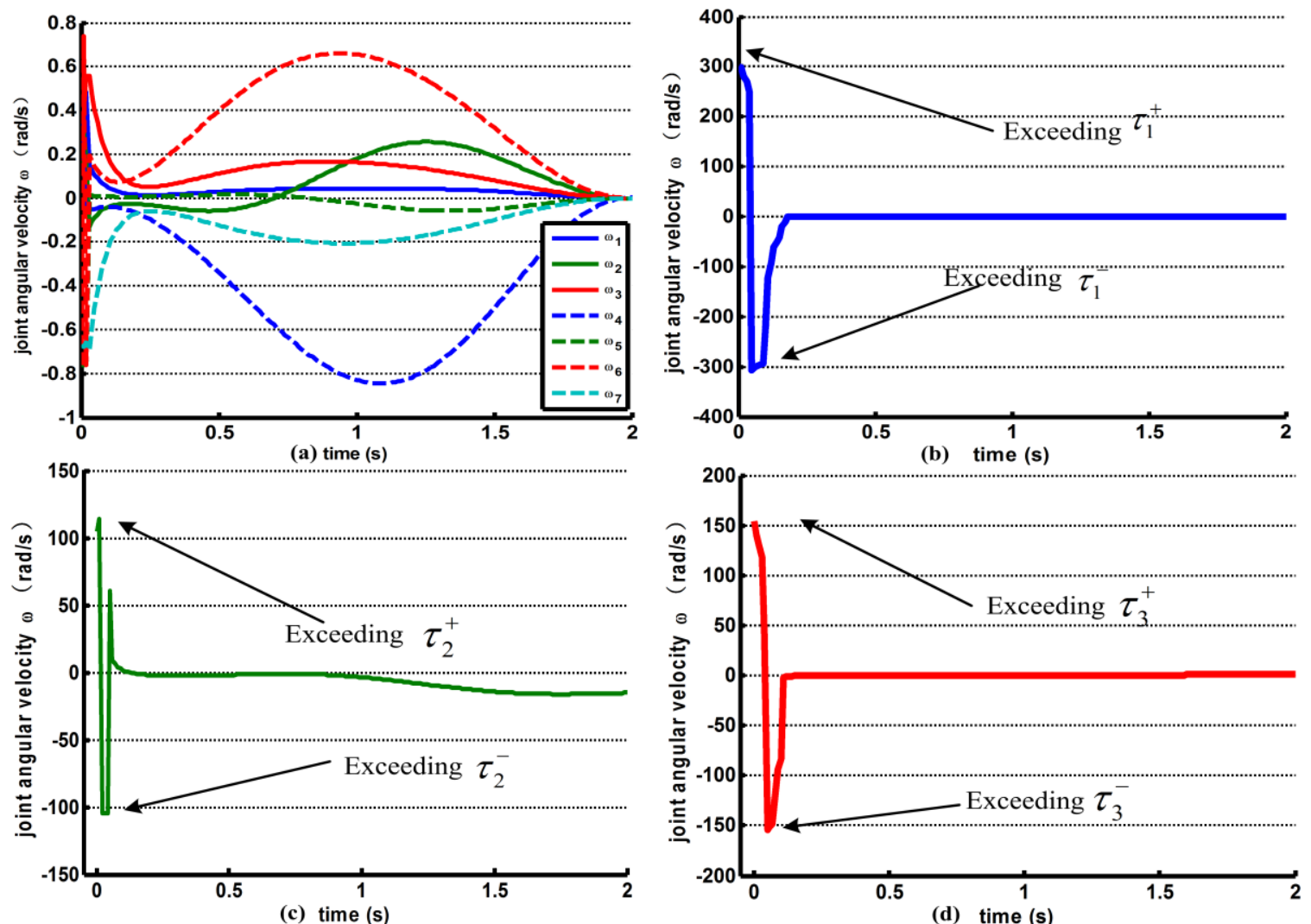

Fig.2. End-effector of the XT arm tracks a straight line path without considering our proposed scheme. (a) Profiles of joint angular velocities. (b) Joint torque within limits. (c) Joint torque within limits. (d) Joint torque within limits. 
Secondly, for comparison and for illustration, the simulation results with both joint torque limits and dual-mode optimal controller being considered are shown in Fig. 3 and Fig. 4. As seen from Fig. 3(a, b), the solution is applicable for the XT-arm working with the straight line path tracking task, because the simulation results shows that the profile position of the end-effector which resolved by the proposed scheme coincide well with the desired trajectory. Compared to the Fig. 1(a, b), Fig. 3(a, b) illustrated that the path tracking task controlled by our proposed scheme is completed more effectively. Furthermore, compared to the Fig. 2(b-d), the joint torque shown in the Fig. 4(b-d) is constrained in their safe limitations. When the torque constraints are activated, the solution space may decrease. Then the dual-mode controller is activated simultaneously. It can be illustrated in the Fig. 5. In this figure, the first mode and second mode are indexed by the value 0 and 1 , respectively.
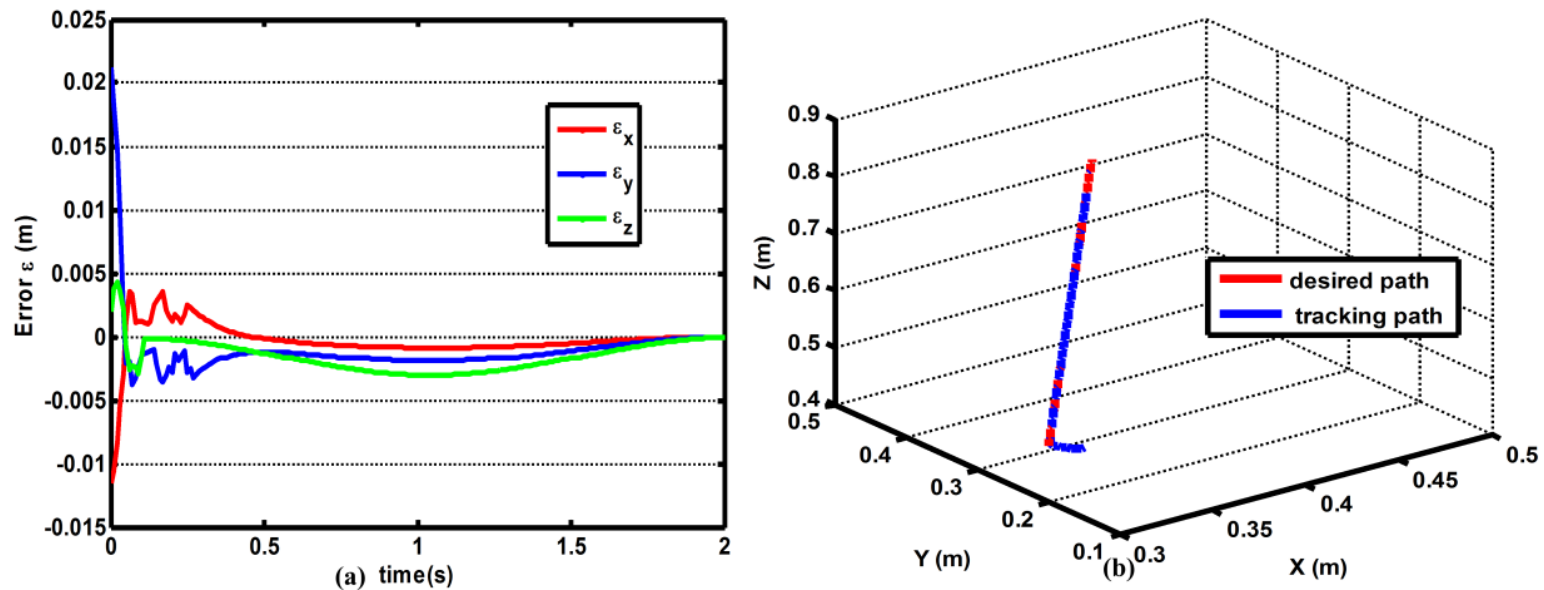

Fig. 3 End-effector of the XT arm tracks a straight line path with both joint physical limits in torque level being considered, where the joint torque is limited in their safe variation range. (a) Simulated End-effector of the XT arm trajectory in Cartesian space (b) Profiles of joint angular velocity.
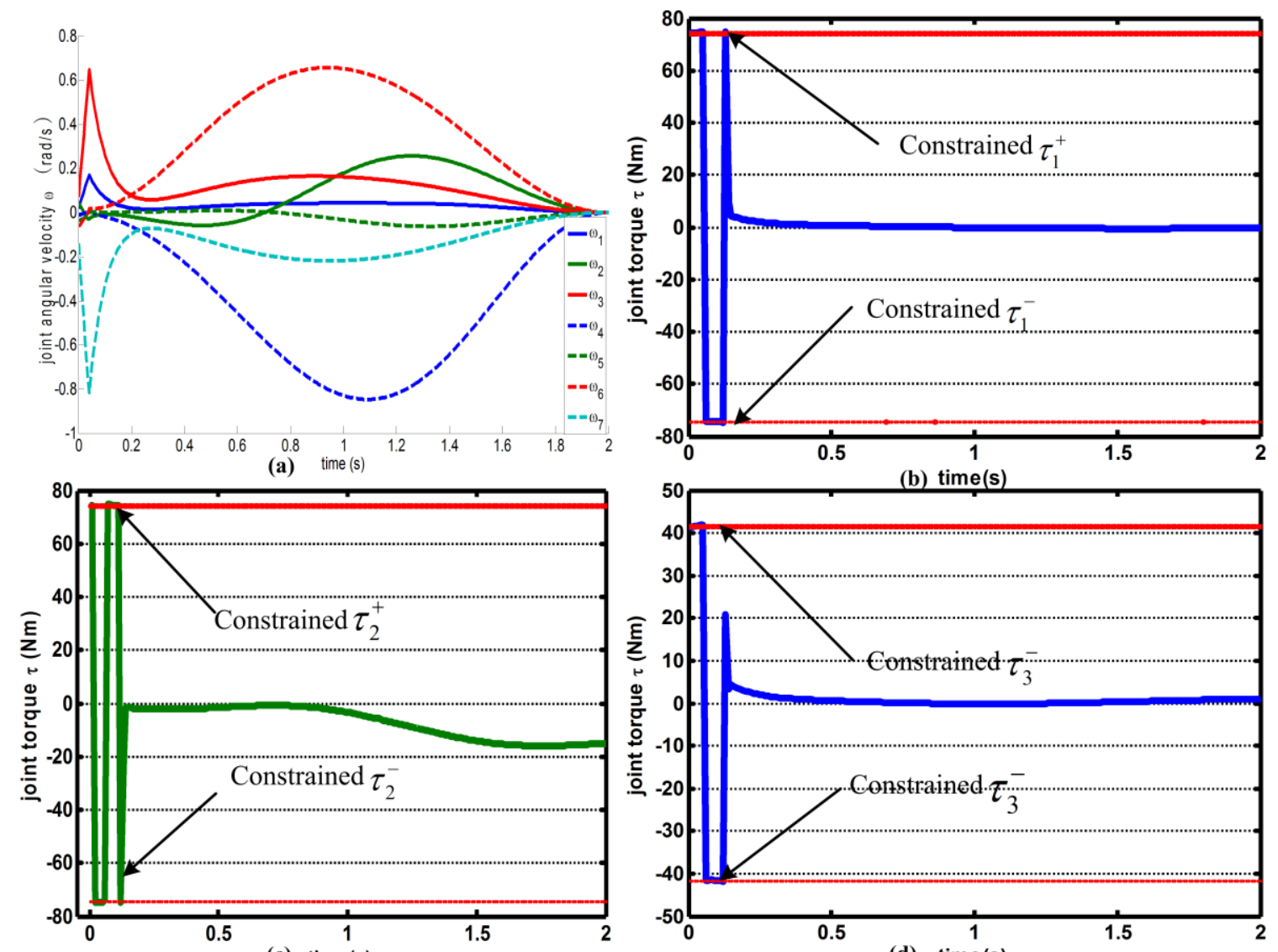

(c) time(s)

(d) time(s)

Fig. 4 XT arm end-effector tracks a straight line path with both joint physical limits in torque level being considered, where the joint torque is limited in their safe variation range. (a) Profiles of first joint torque variation. (b) Profiles of second joint torque variation. (c) Profiles of third joint torque variation. (d) Positioning error of the end-effector. 


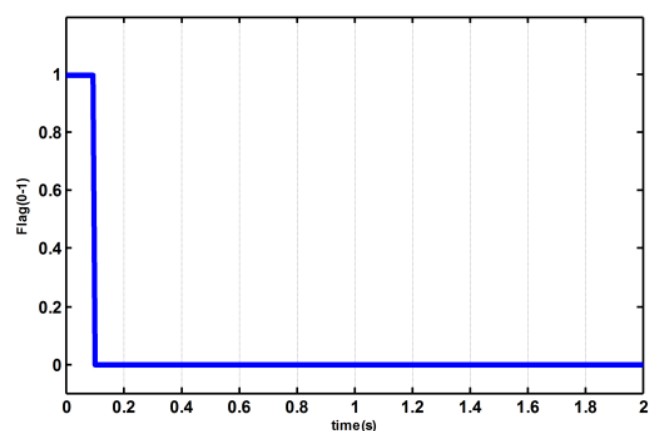

Fig.5 Flag of the mode switch

\section{B. Circular path tracking}

In this section, the tracking task for the XT arm is a circular path. The $\mathrm{X}$-axis and $\mathrm{Y}$-axis position functions of the circular path are

$$
\left\{\begin{array}{l}
x=0.7, \forall t \in[0, T] \\
y=\gamma^{*} \sin \left(\varphi+\cos ^{2}\left(\frac{\pi t}{2 T}\right)\right)+b, \forall t \in[0, T] \\
z=\gamma^{*} \cos \left(\varphi+\cos ^{2}\left(\frac{\pi t}{2 T}\right)\right)+a, \forall t \in[0, T]
\end{array}\right.
$$

Where $\gamma=0.3$ denotes the radius of circular path, $a=0.3$ and $b=0$ denote the coordinate of circle center, $\varphi=\arctan \left(\frac{Y_{0}}{Z_{0}}\right)$, with $\left(X_{0}, Y_{0}, Z_{0}\right)$ denoting the coordinate of the desired path's initial position. Specifically, the tracking task duration time $T=5 \mathrm{~s}$ and the interpolation time is set as $0.01 \mathrm{~s}$ (e.g. $10 \mathrm{~ms}$ ). For the computer simulation, the initial point of the desired path is set as $\left(\begin{array}{lll}0.7000 & 0.2121 & 0.5121\end{array}\right)$ and the $\varphi=\pi / 4$. Without loss of generality, the initial position of the end-effector of the robot manipulator is set at the point $(0.7009$, $0.2059,0.5156)$, which does not coincide with the initial position of the desired circular path and its initial joint angle vector as $\theta(0)=[0.2100,0.6900,0.1134,1.3555$, $-0.0816,-2.0457,-0.3432]^{T}$. The redundancy-resolution results with joint torque limits being considered (i.e. $k_{p}=20$ ).

Firstly, from the Fig. 6, we can see that the initial position of the end-effector does not coincide well with the initial position of the desired path. In the engineering application, this setting is reasonable, because the mechanical error and the actuator error always exist. Because the control law is set in our scheme, the practical trajectory of the end-effector can converge to the desired path quickly. The Fig. 6(a) shows simulated results that the proposed scheme is neglect. We can see that such a solution may not smooth (e.g. end-effector vibration). It can be illustrated by the Fig. 6(b), which shows that the end-effector's position in the X-direction is not stable to converge to the desired path. As for a comparison, the end-effector tracking the desired path with the proposed scheme, Fig. 6(c) shows that the tracking path converged to the desired path effectively. From the Fig. 6(d), we can see that the end-effector's position converge to the desired path in the Xdirection is smoother than that the scheme without considers the torque constraints.
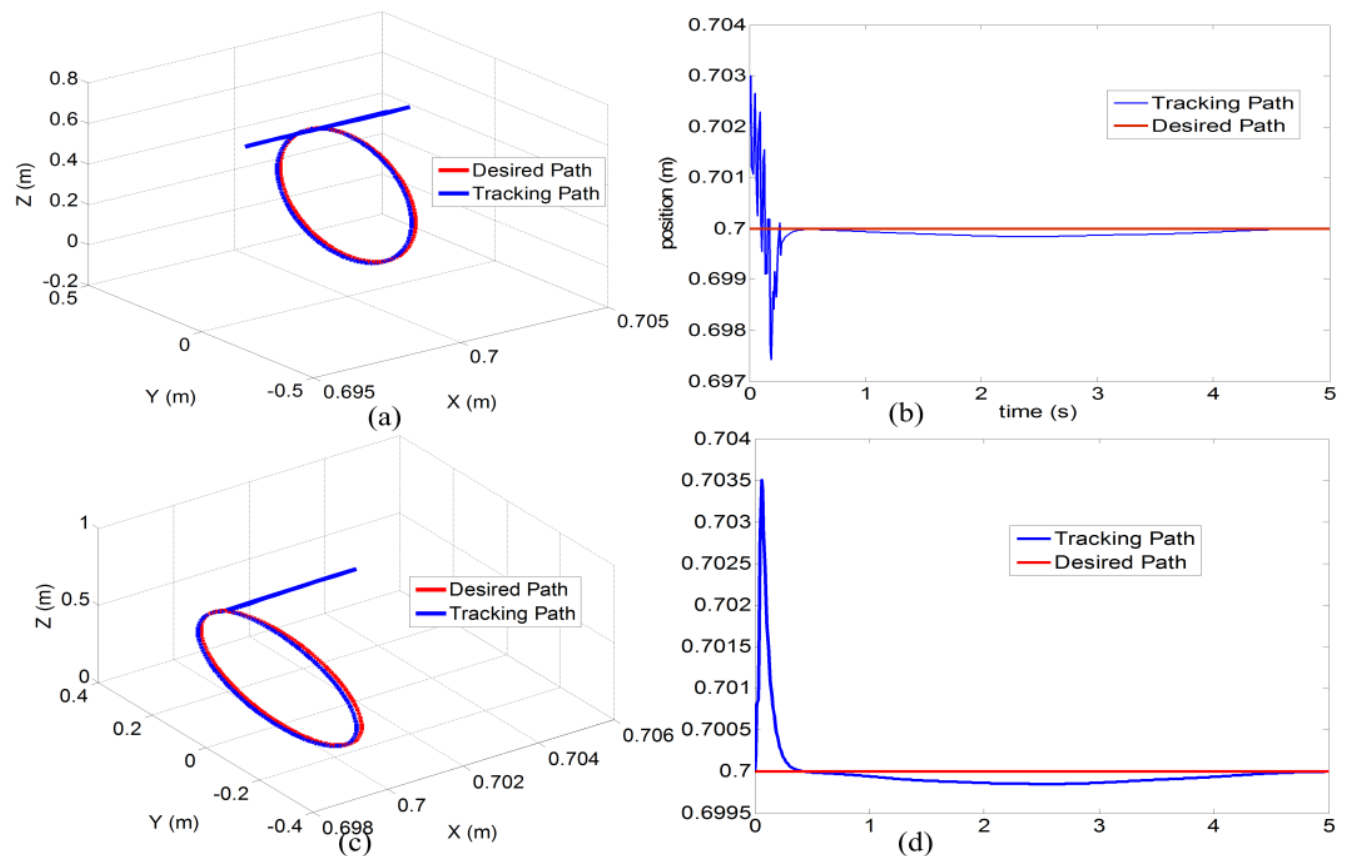

Fig. 6 XT arm end-effector tracks a path of circular path (a) Simulated XT arm manipulator with joint physical limits considered but without considering the constraint in the torque level. (b) The robot tracking path in the X-direction when without considered the torque constraint. (c) Simulated XT arm manipulator with both joint physical limits in torque level. (d) The robot tracking path in the X-direction when considered the torque constraint.

Secondly, the curve of the joint angular velocity variation with proposed scheme is shown in the Fig. 7(a), the end-effector tracking error is shown in the Fig. 4(b). It can be concluded from these figures that the tracking path is sufficiently close to the desired circular path. The simulated results for the arm controlled by our online scheme are shown 
in Fig. 4(c)-(f). As aforementioned, we plot the first three joint torque variation curves for presenting the simulated results. These simulated results illustrate that, by applying the proposed

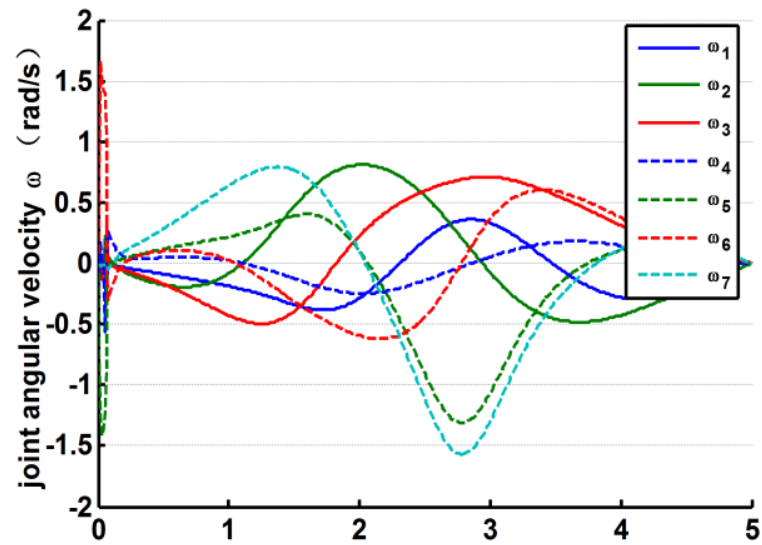

(a) time (s)

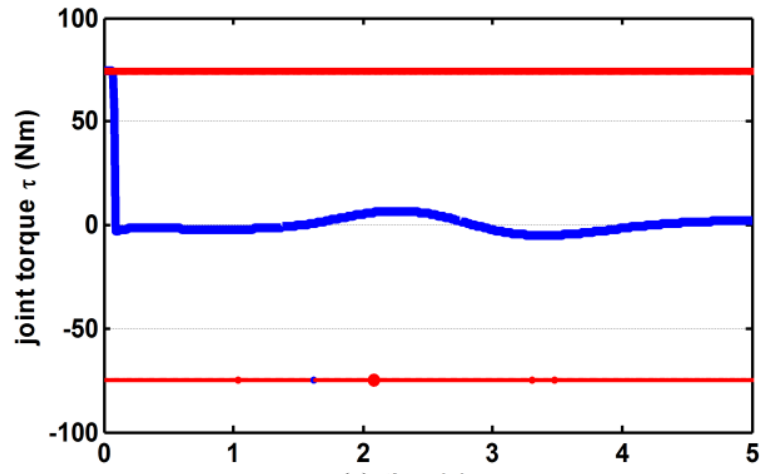

(c) time(s)

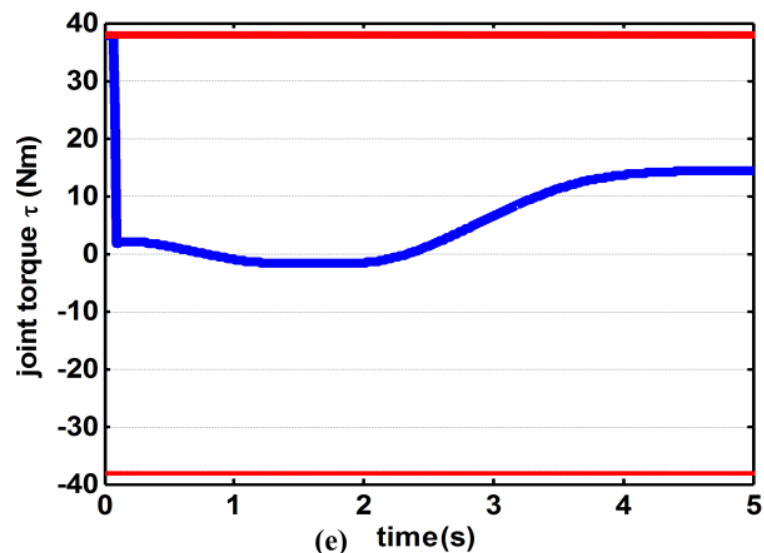

scheme (22-26) to the XT-arm, all joint variables (e.g. joint-velocity and joint torque) are kept within their limited ranges.

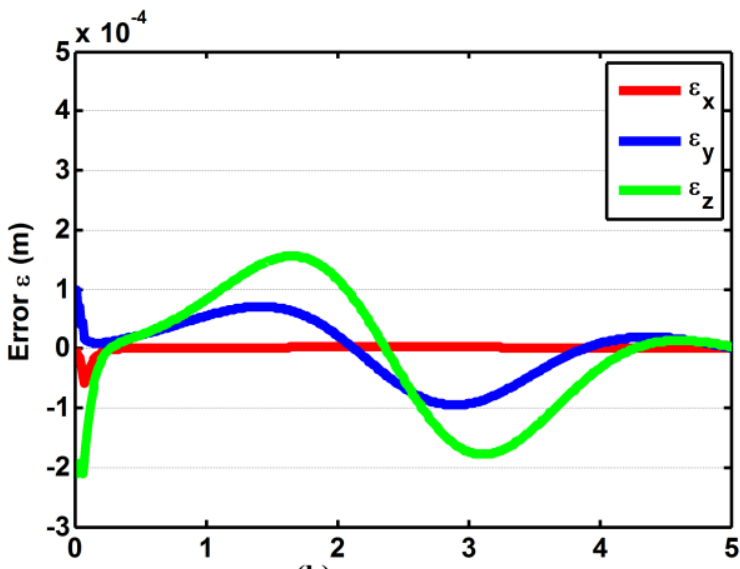

(b) time(s)

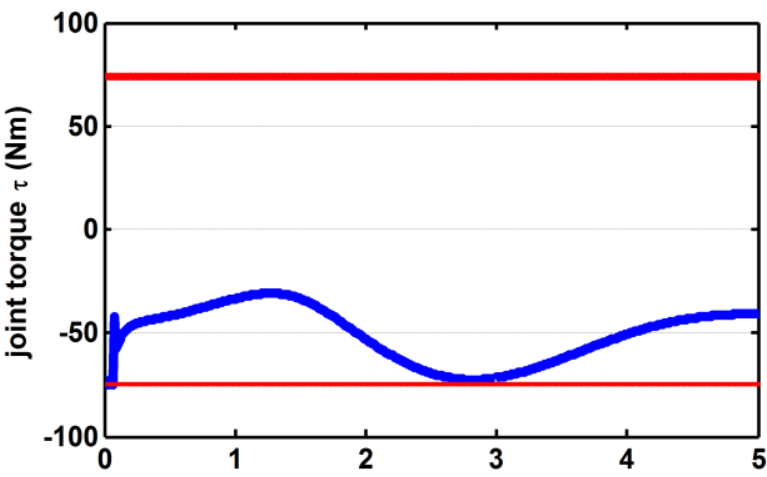

(d) $\operatorname{time}(\mathbf{s})$

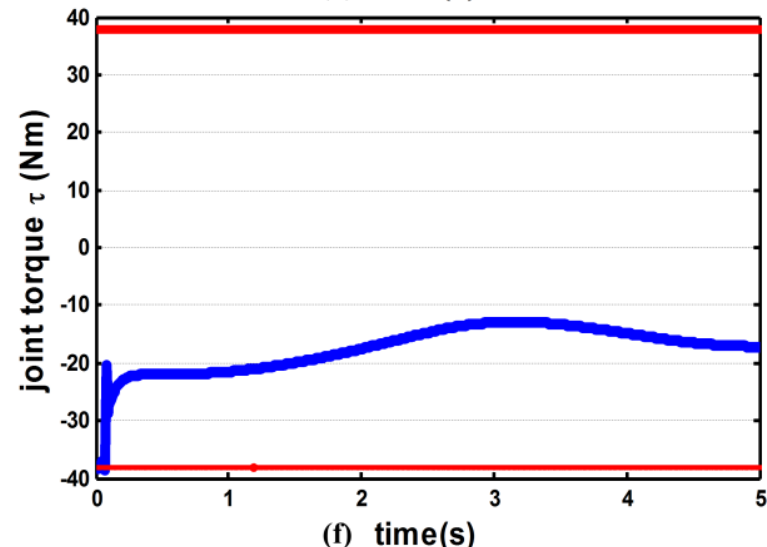

(f) $\operatorname{time}(\mathrm{s})$

Fig. 7 XT Fig. 7 Arm end-effector tracks a circular line path with both joint physical limits in torque level being considered, where the joint torque is limited in their safe variation range. (a) Profiles of joint angular velocity. (b) Positioning error of the end-effector. (c) Profiles of first joint torque variation. (d) Profiles of second joint torque variation. (e) Profiles of third joint torque variation. (f) Profiles of forth joint torque variation.

\section{Solver comparisons}

To verify the efficiency of the proposed Knitro based Solver, the GNN and ZNN for QP proposed in the [32] are used make a comparison. Considering the same configuration of the computer, the simulation is conducted by MATLAB 7.1, performed on a personal digital computer with a Pentium IV 3.20 GHz CPU, 1-GB memory, and Windows7 Professional operating system. The path tracking task of the three-link planar arm is used to make a comparison based on the simulation results. We compared from the views of the computation time and the accuracy. The compared results are shown in the table III.
Table III Comparison Results of Three-Link Planar Arm Simulation

$\begin{array}{ccc} & \text { Computation Time }(s) & \text { Accuracy }(m) \\ G N N & 2.4 \times 10^{-4} & 1.1 \times 10^{-4} \\ \text { ZNN } & 1.1 \times 10^{-4} & 7.0 \times 10^{-7} \\ \text { Knitro } & 5.2 \times 10^{-5} & 4.0 \times 10^{-6}\end{array}$

In this table, the accuracy of our proposed solver is $4.0 \times$ $10^{-6} \mathrm{~m}$. It is in the range between the ZNN and GNN. But the computation time of our proposed method is $5.2 \times 10^{-5} \mathrm{~s}$ which is less than others an order of magnitude. Then our Knitro based solver could be used to online optimization control when we set the interpolation period as $1 \times 10^{-3} \mathrm{~s}$. In the field of the arm manipulation, the magnitude of the end-effector vibration may 
reach $10^{-6} \mathrm{~m}$ or $10^{-7} \mathrm{~m}$. Relatively, the interface of the Knitro is implemented using $\mathrm{C}$ language; it can be integrated to the engineering application more conveniently than the network based solvers. Therefore, the Knitro solver may more suitable than others on the prospective of the computation time and accuracy.

\section{HARDWARE EXPERIMENT VERIFICATION}

To verify the proposed scheme in a physical arm manipulator, we perform the scheme (22-26) with $k_{p}=15$ on the 7-DOF arm manipulator of the XT. The path tracking task is designed to complete the valve operation task of the competition.

The whole robot hardware system is composed of a controller and a manipulator. The robot controller is an embedded computer with a Pentium 1.6-GHz CPU, 1-GB DDR2 memory, and a Linux with RTAI real-time operating system, which controls the robot joint using a CAN bus controller. The frame structure of the hardware system could be shown in the Fig. 8. Fig. 9 shows the XT robot with the dual-arm. The upper and lower limits of the joint angles are same to the parameters which we used in the simulation work. Two straight line paths are shown in the following experiment results: the horizontal line and the vertical line. The vertical tracking path which start from the point $[0.44,0.12,0] \mathrm{m}$ to the point $[0.44,-0.020,0] m$. The task duration is $2.0 \mathrm{~s}$. The experimental results are shown in the Fig. 10.

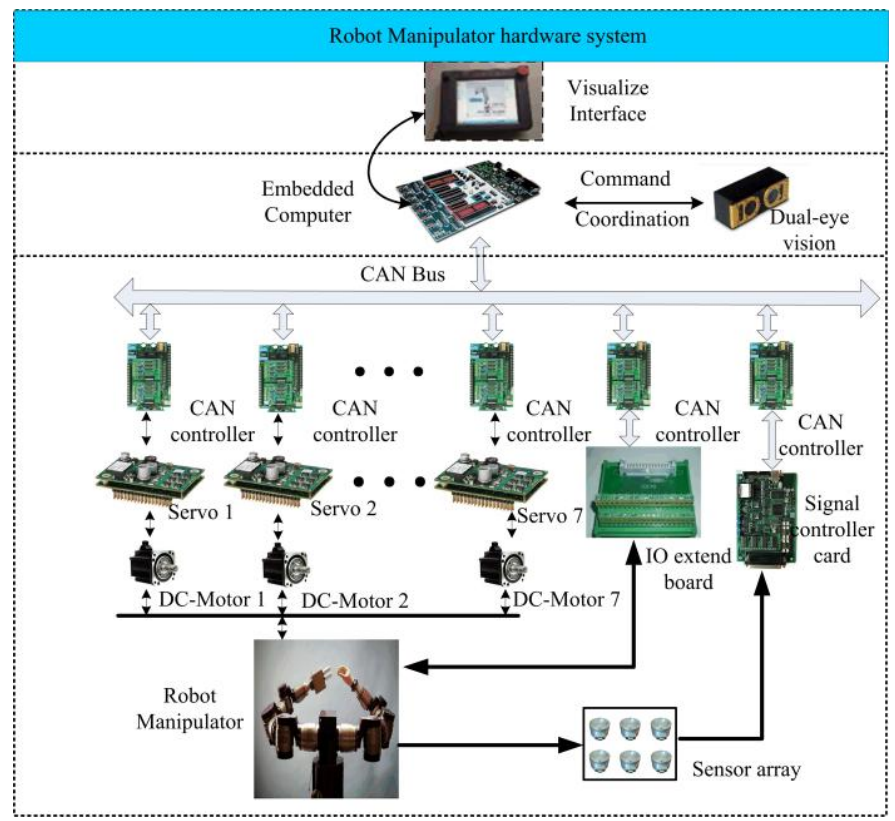

Fig. 8 Hardware structure of the robot control system
The snapshots of the task execution when the robot tracks the straight-line path are shown in Fig. 10(a)-(d), which demonstrates the task could be executed well. In addition, to present the results clearly, we show the tracking path straight line (PTSL) of the end-effector in the Fig. 7(e), which compared with a standard straight line (SSL) drew by a standard ruler. In addition, the horizontal line and the vertical line path tracking results is shown in the Fig. 7(f). The accuracy of the tracking task is illustrated by analysis of the error curve shown in the Fig. 7 (g) and (f), which is shown the maximal positioning error is less than $1.5 \times 10^{-4} \mathrm{~m}$. It illustrates that the tracking path of the end-effector is sufficiently close to the desired straight-line path, namely, the tracking task can be completed well. Its corresponding computation time is shown in the Fig. 7(h), (i), respectively. The maximal computation time cost during over all task kinematics resolution is less than $8 \times 10^{-3} \mathrm{~s}$, which can ensure the redundancy-resolution computed online.

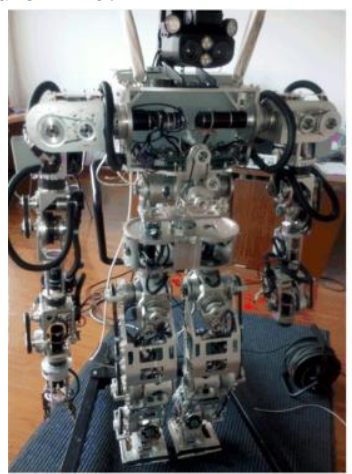

(a)

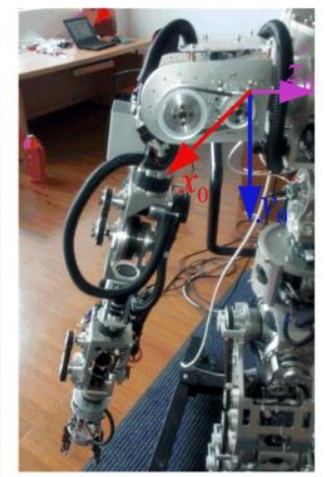

(b)
Fig. 97-DOF humanoid robot manipulator 


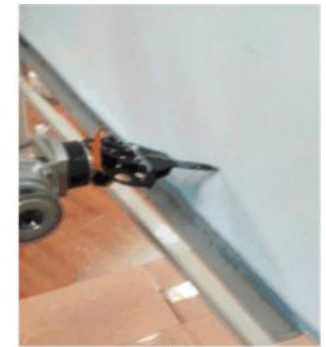

(a)

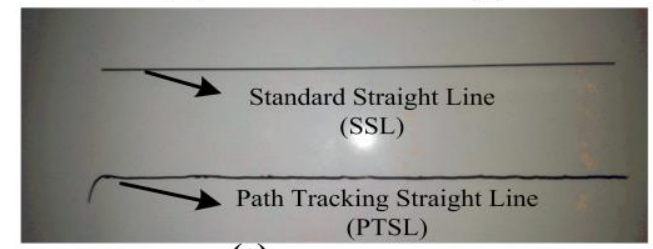

(e)
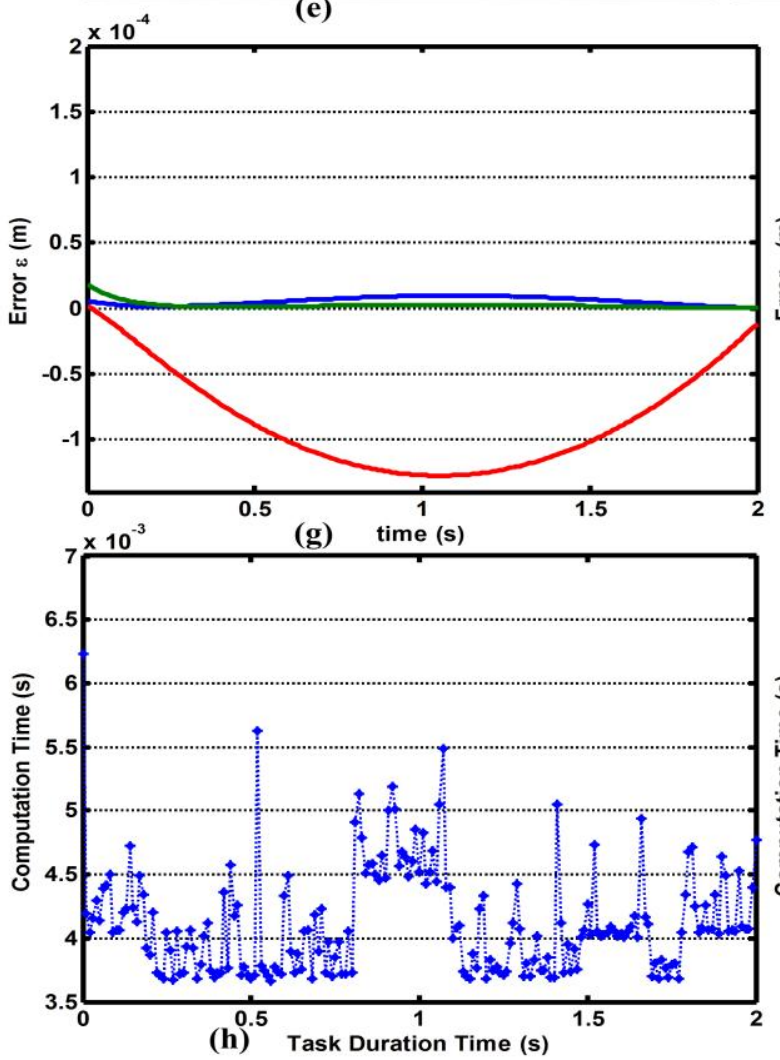

VI. CONCLUSION

In this paper, we have proposed and investigated a novel online method to resolve the kinematics of physical-constrained redundant manipulators in the torque level. The constraint model is extended to the torque level. The dual-mode optimal controller is integrated to solve the problem of the decreasing solution space. Then the Knitro-Based solver is first used to solve the QP which is converted from our online scheme. Both the computer simulations and physical robot experiments have demonstrated the effectiveness, physical realizability, accuracy, and safety of the proposed online scheme for torque constrained redundant robot manipulators.

\section{ACKNOWLEDGMENT}

This research has been supported by National Science and Technology Support Program（No.2015BAK06B02）, project

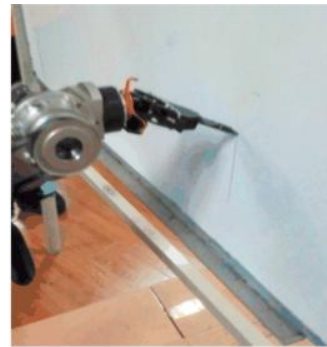

(c)

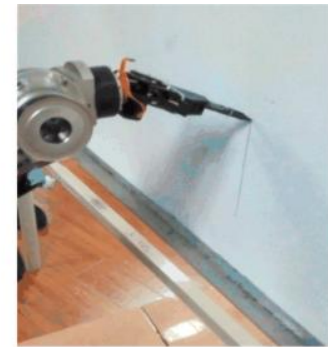

(d)
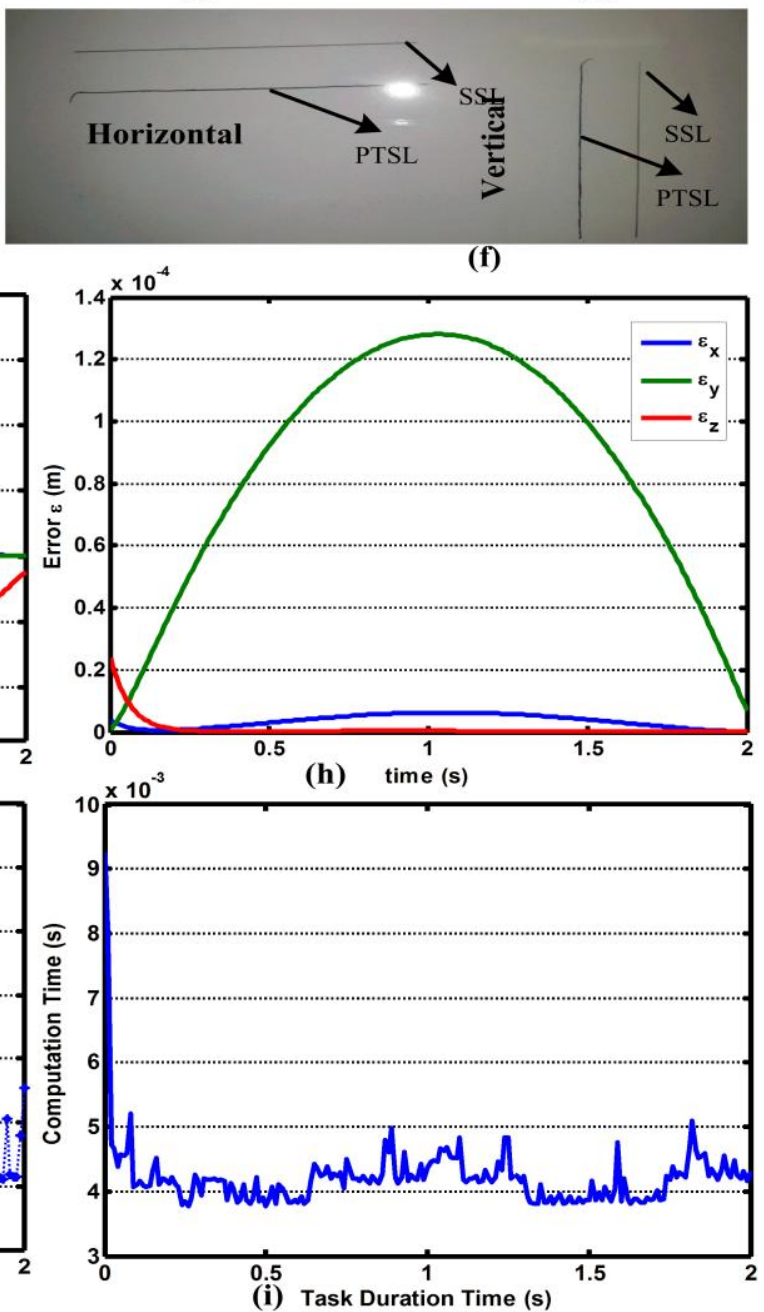

of Science and Technology Support Plan of Jiansu province (No. BE2013003) and Project of the National Science Foundation of China (No. 51405470) and (No. 51405469). Thanks are extended to our colleagues at Lab for cooperation on project of dual-arm redundant robot. Friends and former colleagues at USTC are also acknowledged for fruitful discussions on robot control and applications.

\section{REFERENCE}

[1] A. Abbaspour, H. Z. Jafari, M. A. A. Hemmat, and etc, "Redundancy Resolution for Singularity Avoidance of Wheeled Mobile Manipulators", Proceedings of the ASME 2014 International Mechanical Engineering Congress and Exposition, pp. 1-10, 2014

[2] A. A. Maciejewski and C. A.Klein, "Obstacle avoidance for kinematically redundant manipulators in dynamically varying environments," Int. J. Robot. Res., vol. 4, no. 3, pp. 109-117, 
1985.

[3] Y. Zhang and J. Wang, "A dual neural network for constrained joint torque optimization of kinematically redundant manipulators," IEEE Trans. Syst., Man, Cybern. B, vol. 32, no. 5, pp. 654-662, Oct. 2002.

[4] A. Liegeois, "Automatic supervisory control of the configuration and behavior of multibody mechanisms," IEEE Trans. Syst., Man, Cybern.,vol. SMC-7, no. 12, pp. 868-871, Dec. 1977.

[5] K. Tchon'“Optimal extended Jacobian inverse kinematics algorithms for robotic manipulators," IEEE Trans. Robot. Vol. 24, no. 6, pp:1440-1445, 2008;

[6] P. H. Chang, "A closed-form solution for inverse kinematics of robot manipulators with redundancy," IEEE $J$. Robot. Autom., vol. RA-3, no. 5,pp. 393-403, Oct. 1987.

[7] L. Sciavicco and B. Siciliano, "A solution algorithm to the inverse kinematic problem for redundant manipulators," IEEE J. Robot. Autom., vol. 4,no. 4, pp. 403-410, Aug. 1988.

[8] H. Seraji, "Configuration control of redundant manipulator: Theory and implementation," IEEE Trans. Robot. Autom., vol. 5, no. 4, pp. 472-490, Aug. 1989.

[9] T. F. Chan and R.V. Dubey, "A weighted least-norm solution based scheme for avoiding joint limits for redundant joint manipulators," IEEE Trans. Robot. Autom., vol. 11, no. 2, pp. 286-292, Apr. 1995.

[10] A. A. Maciejewski and C. A.Klein, "Obstacle avoidance for kinematically redundant manipulators in dynamically varying environments," Int. J. Robot. Res., vol. 4, no. 3, pp. 109-117, 1985.

[11] J. Baillieul, "Avoiding obstacles and resolving kinematic redundancy," in Proc. IEEE Int. Conf. Robot. Autom., 1986, pp. 1698-1704.

[12] M. John, K. C. Suh, "Redundancy Resolution of Manipulators through Torque Optimization", IEEE Journal of Robotics and Automation, vol. RA-3, NO. 4, 1987.

[13] J. Xiang, C. Zhong, and W.Wei, "General-weighted least-norm control for redundant manipulators," IEEE Trans. Robot., vol. 26, no. 4, pp. 660-669, Aug. 2010.

[14] J. Xiang, C. Zhong, and W.Wei, "A Varied Weights Method for the Kinematic Control of Redundant Manipulators With Multiple Constraints," IEEE Trans. Robot. vol. 28, no. 2, pp. 330-339, April 2012.

[15] Y. Zhang, D. Guo, B. Cai, "Remedy scheme and theoretical analysis of joint-angle drift phenomenon for redundant robot manipulators", Robot. Comput. -Integr. Manuf., vol. 27, no. 4, 860-869, 2011.

[16]Y. Zhang, J. Yin, and B. Cai, "Infinity-norm acceleration minimization of robotic redundant manipulators using the LVI-based primal-dual neural network," Robot. Comput. -Integr. Manuf., vol. 25, no. 2, pp. 358-365, Apr. 2009.

[17] B. Cai and Y. Zhang, "Bi-criteria optimal control of redundant robot manipulators using LVI-based primal-dual neural network," Optim. Control Appl. Methods, vol. 31, no. 3, pp. 213-229, May/Jun. 2010.

[18] Z. Zhang, Y. Zhang, "Variable Joint-Velocity Limits of Redundant Robot Manipulators Handled by Quadratic Programming", IEEE/ASME Trans Mechatronics, Vol 8, No. 2,
674-686, 2013

[19] D. Guo, Y. Zhang, "Acceleration-Level Inequality-Based MAN Scheme for Obstacle Avoidance of Redundant Robot Manipulators", IEEE Trans. Ind. Electron., vol. 61, No. 12, 6903-6914, 2014.

[20] Z. Zhang, Y. Zhang, "Acceleration-Level Cyclic-Motion Generation of Constrained Redundant Robots Tracking Different Paths", IEEE Trans. Syst., Man, Cybern. B. Vol. 42, No. 4:1257-1269, 2012.

[21] J. Baillieul, "Kinematic programming alternatives for redundant manipulators," In: Proceedings of IEEE international conference of robotics and automation, vol. 1, pp. 722-728, 1985.

[22] T. Shamir, Y. Yomdin, "Repeatability of redundant manipulators: mathematical solution of the problem," IEEE Trans. Autom. Control, 33(11):1004-1009, 1988.

[23] Y. Zhang, Y. Yang, N. Tan, and B. Cai, "Zhang neural network solving for time-varying full-rank matrix Moore-Penrose inverse," Computing, vol. 92, no. 2, pp. 97-121, Jun. 2011.

[24] B. Cai and Y. Zhang, "Different-Level Redundancy-Resolution and Its Equivalent Relationship Analysis for Robot Manipulators Using Gradient-Descent and Zhang et al.'s Neural-Dynamic Methods", IEEE Trans. Ind. Electron.,, vol. 59, no. 8, 3146-3155, Aug. 2012.

[25] C. W. Wampler, "Manipulator inverse kinematic solutions based on vector formulations and damped least-squares methods," IEEE Trans. Syst., Man, Cybern., vol. SMC-16, no. 1, pp. 93-101, Jan. 1986.

[26] F. T. Cheng, T. H. Chen, Y. Y. Sun, "Resolving Manipulator Redundancy Under Inequality Constraints", IEEE Trans. Robot. Autom., V1L. 10, no. 1, Feb, 1994.

[27] D. G. Luenberger, Linear and Nonlinear Programming. Addison-Wesley Publishing Company, second edition, 1984.

[28] R. H. Byrd, J. Nocedal, and R. A. Waltz, "KNITRO: An Integrated Package for Nonlinear Optimization," in Large-Scale Nonlinear Optimization, G. di Pillo and M. Roma, Ed., Springer-Verlag, pp. 35-59, 2006. 